\title{
Oncogenic driver genes and tumor microenvironment determine the type of liver cancer
}

Gang Wang ${ }^{1,2}$, Qian Wang ${ }^{2,3}$, Ning Liang $\mathbb{1}^{4}$, Hongyuan Xue ${ }^{5}$, Tao Yang ${ }^{6}$, Xuguang Chen ${ }^{7}$, Zhaoyan Qiu ${ }^{8}$, Chao Zeng ${ }^{9}$, Tao Sun ${ }^{10}$, Weitang Yuan ${ }^{3}$, Chaoxu Liu ${ }^{5,11}$, Zhangqian Chen ${ }^{12,13}$ and Xianli He ${ }^{2}$

\begin{abstract}
Primary liver cancer (PLC) may be mainly classified as the following four types: hepatocellular carcinoma (HCC), intrahepatic cholangiocarcinoma (ICC), hepatoblastoma (HB), and combined hepatocellular carcinoma and intrahepatic cholangiocarcinoma (CHCC-ICC). The majority of PLC develops in the background of tumor microenvironment, such as inflammatory microenvironments caused by viral hepatitis, alcoholic or nonalcoholic steatohepatitis, carbon tetrachloride (CCl $)$, 3,5-diethoxycarbonyl-1,4-dihydrocollidine (DDC), and necroptosisassociated hepatic cytokine microenvironment caused by necroptosis of hepatocytes. However, the impact of different types of microenvironments on the phenotypes of PLC generated by distinct oncogenes is still unclear. In addition, the cell origin of different liver cancers have not been clarified, as far as we know. Recent researches show that mature hepatocytes retain phenotypic plasticity to differentiate into cholangiocytes. More importantly, our results initially demonstrated that HCC, ICC, and CHCC-ICC could originate from mature hepatocytes rather than liver progenitor cells (LPCS), hepatic stellate cells (HSCs) and cholangiocytes in AKT-driven, AKT/NICD-driven and AKT/CATdriven mouse PLC models respectively by using hydrodynamic transfection methodology. Therefore, liver tumors originated from mature hepatocytes embody a wide spectrum of phenotypes from HCC to CC, possibly including CHCC-ICC and HB. However, the underlying mechanism determining the cancer phenotype of liver tumors has yet to be delineated. In this review, we will provide a summary of the possible mechanisms for directing the cancer phenotype of liver tumors (i.e., ICC, HCC, and CHCC-ICC) in terms of oncogenic driver genes and tumor microenvironment. Moreover, this study initially revealed the cell origin of different types of liver cancer.
\end{abstract}

\section{Facts}

- Liver tumor phenotype is defined by a combination of driving oncogenes but also the types of tumor

Correspondence: Chaoxu Liu (chaoxuliu@yahoo.com) or

Zhangqian Chen (Chenzq1@fmmu.edu.cn) or Xianli He (hexianli999@126.com)

${ }^{1}$ Department of General Surgery, The 74th Group Army Hospital, Guangzhou

510220, China

${ }^{2}$ Department of General Surgery, Tangdu Hospital, Air Force Military Medical

University, Xi'an 710032 Shaanxi, China

Full list of author information is available at the end of the article

These authors contributed equally: Gang Wang, Qian Wang, Ning Liang,

Hongyuan Xue

Edited by $\mathrm{Q}$. Chen microenvironments.

- Necroptosis-associated hepatic microenvironment facilitates formation of ICC, whereas apoptosisassociated hepatic microenvironment promotes formation of $\mathrm{HCC}$.

- HCC, ICC, and cHCC-ICC could originate from mature hepatocytes in mouse models by using hydrodynamic transfection methodology.

\section{Open questions}

- In addition to mature hepatocytes, it is not clear whether liver cancer could originate from hepatic 
progenitor cells, hepatic stellate cells and bile duct cells.

- It's uncertain whether the formation of cHCC-ICC was jointly caused by necroptosis environment and apoptosis environment, which needs to be verified in the future.

- In the course of chemoembolization therapy for patients with HCC, a phenotypic transition from HCC to ICC was observed. The possible mechanism may lie in the necroptosis-associated hepatic microenvironment caused by chemoembolization therapy, suggesting the cell environment may directly affect the choice of treatment methods.

\section{Introduction}

Primary liver cancer (PLC) is the fifth most prevalent cancer and the third common cause of cancer-related mortality worldwide ${ }^{1}$. PLC is insensitive to various treatments, which could be partly explained by its wide genetic variations, reflecting in the diverse phenotypes and histological characters. PLC may be mainly classified as the following four types: hepatocellular carcinoma (HCC), intrahepatic cholangiocarcinoma (ICC), hepatoblastoma $(\mathrm{HB})$, and combined $\mathrm{HCC}$ and intrahepatic cholangiocarcinoma (cHCC-ICC) ${ }^{2}$. cHCC-ICC, as an intermediate variant of PLC, has attracted more and more attention in recent years. cHCC-ICC is described as demonstrating histologic features of both hepatocellular and biliary epithelial differentiation ${ }^{3}$. It is a rare primary liver malignancy, accounting for 1-14.2\% of cases. However, the cell origin of PLC is still controversial.

From morphological and pathological perspectives, HCC and ICC were previously considered to originate from hepatocytes and cholangiocytes, respectively ${ }^{4}$. In addition, some subtypes of HCC with fetal hepatoblasts features are thought to arise from hepatic progenitor cells, which may differentiate into hepatocytes and bile duct epithelial cells under certain stimuli ${ }^{5}$. Generally, the cell origin of PLC may be derived from the following four types of cells: hepatocytes, cholangiocytes, hepatoblasts, and liver stem/progenitor cells. However, the cell origin of PLC and the underlying mechanism for the phenotypic determination remains unclear. Recently, some wellestablished lineage-tracing mouse experiments have demonstrated that HCC or ICC originates from mature hepatocytes rather than liver progenitor cells (LPCs), hepatic stellate cells (HSCs), and cholangiocytes. For example, one study showed that ICC could originate from hepatocytes in mice when the PI3K-AKT and Notch pathways were coactivated ${ }^{6}$. On a similar note, $\mathrm{Mu}$ et al. demonstrated that hepatocytes represent the cell of origin for HCC in mice. Moreover, for the subtype of HCC with a progenitor signature, it does reflect progenitor origin, but dedifferentiation of hepatocyte-derived tumor cells ${ }^{7}$.
Therefore, liver tumors originated from mature hepatocytes consist of a wide spectrum of phenotypes from HCC to $\mathrm{CC}$, possibly encompassing $\mathrm{CHCC}-\mathrm{ICC}$ and $\mathrm{HB}^{4}$.

In this review, we will summarize the potential mechanisms for determining the cancer phenotype of hepatocyte-derived mouse liver tumors, including ICC, $\mathrm{HCC}$, and cHCC-ICC, in terms of oncogenic driver genes and tumor microenvironment by combining our previous work and the latest research progress. More importantly, it may help us screen of innovative therapeutic approaches against this deadly malignancy in the future.

\section{Regulatory molecules and tumor microenvironment that commit ICC formation}

Previous studies have shown that ICC may originate from the cells lining the bile ducts, biliary duct cells (BDCs) or liver stem/progenitor cells ${ }^{8}$. Nevertheless, recent studies have demonstrated that mature hepatocytes possess a capacity for cholangiocytes transdifferentiation under certain conditions ${ }^{9}$. For example, Nishikawa et al. ${ }^{10}$ found that cultured hepatocytes expressed several bile duct markers including cytokeratin (CK) 19 in a threedimensional organoid culture system, which containing insulin and epidermal growth factor. Likewise, Michalopoulos et al. ${ }^{11}$ showed that hepatocytes can transdifferentiate into BDCs and help repair the damaged biliary epithelium when its proliferative capacity is being compromised. Moreover, a recent study also showed that mature hepatocytes exhibited the bile duct-like phenotype after chronic liver injury both in vivo and in vitro ${ }^{9}$. In addition, the notion that cell origin of ICC is mature hepatocytes was subsequently confirmed in another chemically induced ICC mouse model ${ }^{12}$, as well as a study by electroporating oncogenic transposon plasmids into the left liver lobe of mice ${ }^{13}$. Recent studies have shown a significant difference between the primary BDCs and the hepatocytes transdifferentiated BDCs. Morphologically, these hepatocytes transdifferentiated BDCs are not mature cholangiocytes with reserve for hepatocyte differentiation. Functionally, hepatocyte-derived ductules are not conducive to bile drainage. Importantly, a recent study demonstrated that these hepatocytes transdifferentiated BDCs are transcriptionally distinct from the primary BDCs as shown by RNA-sequencing analysis and ultrastructural analysis. Interestingly, these hepatocytes transdifferentiated BDCs keep their origin memory and could revert back to hepatocytes upon cessation of injury, which reflecting an adaptive injury escape mechanism ${ }^{14}$. Mechanistically, TGF $\beta$ signaling has been identified associated with the formation of the transdifferentiated BDCs from hepatocytes.

In our previous research, we applied hydrodynamic tail vein injection of hemagglutinin (HA) tagged AKT and NICD plasmid (AKT/NICD), along with Sleeping Beauty 


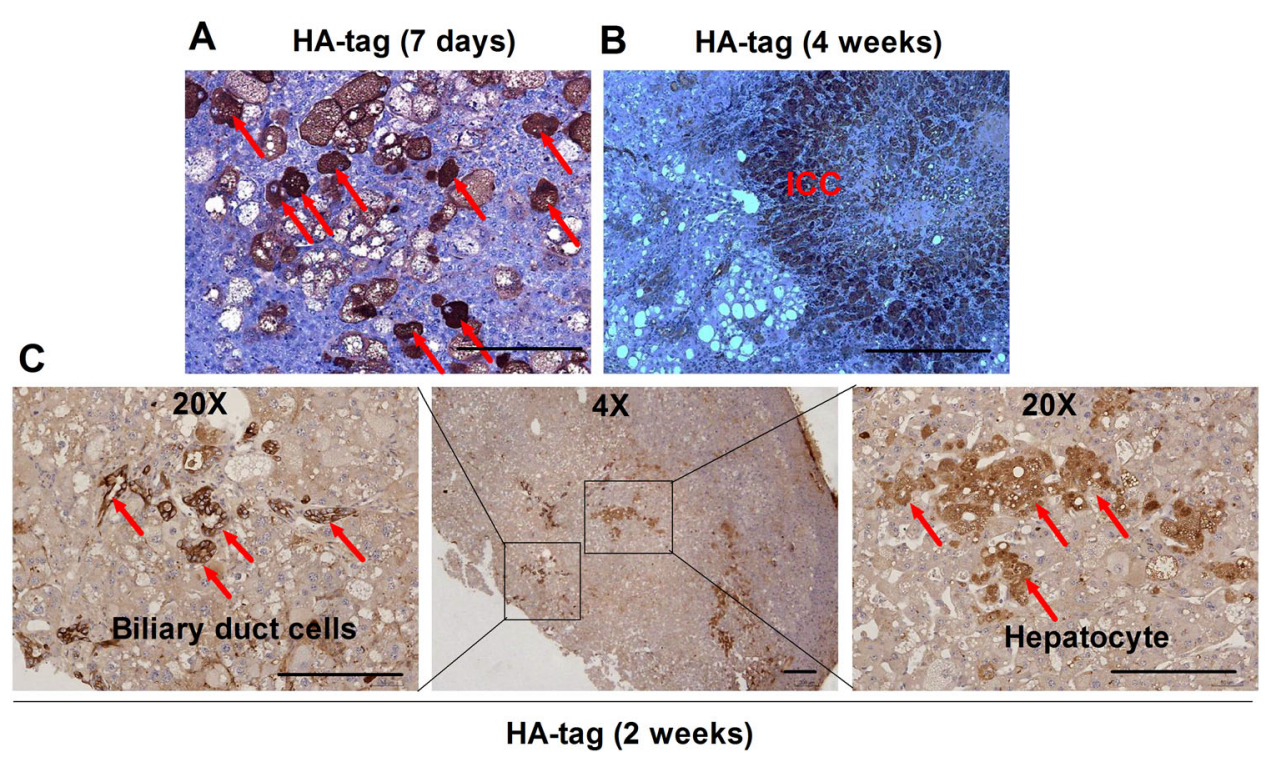

Fig. 1 ICC could originate from hepatocytes. a Immunohistochemical staining (IHC) showed that some scattered HA-tag strongly positive hepatocytes were detected in AKT/NICD-injected livers after 7 days. $\mathbf{b} I \mathrm{HC}$ results showed that HA-tag protein was also expressed in ICC tissues after 4 weeks. $\mathbf{c} \mid \mathrm{HC}$ results showed that some HA-stained hepatocytes and biliary duct cells appeared after 2 weeks. ICC: intrahepatic cholangiocarcinoma (scale bars, $50 \mu \mathrm{m})$.

(SB) plasmids into BALB/c mice (6-8 weeks) to initiate ICC development. After 7 days, some scattered HA-tag strongly positive hepatocytes were detected in AKT/ NICD-injected livers (Fig. 1a). After 4 weeks, immunohistochemical results showed that HA-tag protein was also expressed in ICC, indicating that ICC could originate from these HA-tag positive hepatocytes (Fig. 1b) After 2 weeks, we found that some HA-stained hepatocytes and BDCs appeared at the same time, which further proved the hepatocytes transdifferentiation (Fig. 1c). Accordingly, cellular reprogramming- transition from hepatocyte towards a more ICC-like phenotype might be induced by genetic and cellular alterations occurring during tumorigenesis. Based on the latest research, mechanism underlying hepatocyte-derived ICC formation can be summarized as the following aspects (Fig. 2).

\section{Regulatory molecules that commit ICC formation Notch signaling pathway}

Accumulating evidence suggests that the canonical Notch cascade controls hepatocyte-derived ICC formation in mice ${ }^{12,14}$. Notch is a highly conservative signaling pathway that regulates cell proliferation and differentiation and plays an important role in embryonic development and in cell fate determination ${ }^{15}$. By using wellestablished lineage-tracing mouse models, Yanger et al. ${ }^{16}$ report that the activation of Notch is sufficient to reprogram hepatocytes into biliary epithelial cells under injury conditions that provoke a biliary response. Likewise, Fan et al. ${ }^{6}$ and Sekiya et al. ${ }^{12}$ also show that ICC can originate

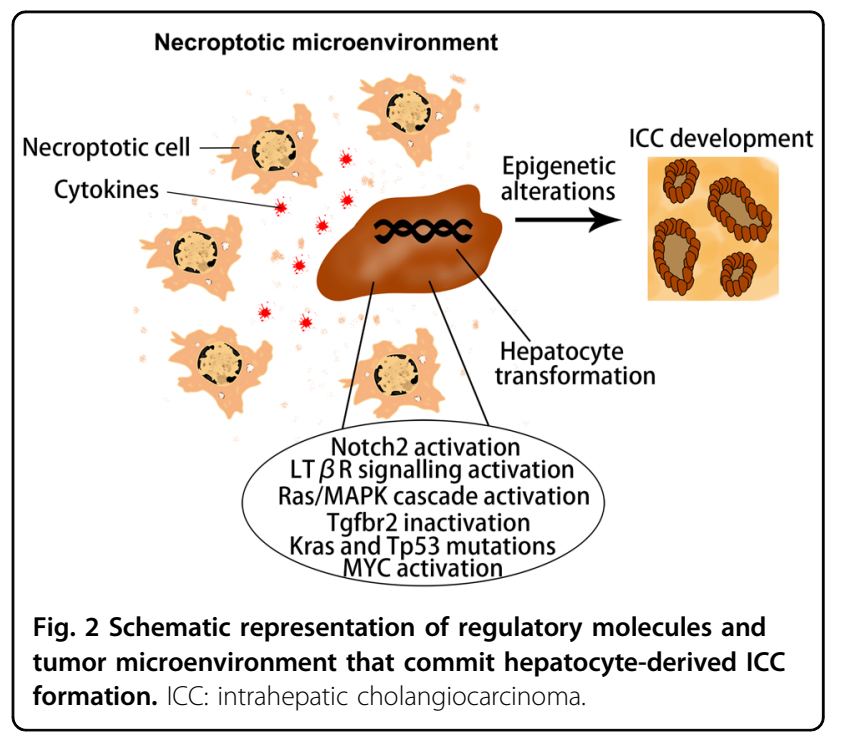

from fully differentiated hepatocytes by using a mouse model of hepatocyte fate tracing. However, which Notch receptor is responsible for hepatocyte-derived ICC formation appears to be more important. Most recently, a new study reveals that Notch2, rather than Notch1, controls hepatocyte-derived ICC formation in mice. In this study, Chen et al. established a murine hepatocyte-derived ICC model by co-expression of AKT and Yap plasmids in mice liver ${ }^{14}$. They demonstrated that deletion of Notch2 skews AKT/Yap-induced ICC pathology towards a more hepatocellular adenoma-like phenotype. 
However, deletion of Notch1 in tumor cells does not affect the histological type ${ }^{14}$. Therefore, endogenous Notch signaling is required for hepatocyte-derived ICC. This finding suggested that Notch2 could serve as a target for treatment of this deadly disease, which has great impact on clinical practice in the foreseeable future.

\section{Kras and Tp53 mutations}

The recent study of Hill et al. ${ }^{17}$ indicates Kras and Tp53 mutations facilitate formation of hepatocyte-derived ICC in the context of chronic liver injury. By using Alb-Cre; $\mathrm{Kras}^{L S L-G 12 D} ; T p 53^{f / f}$ transgenic mice that targeting Kras and Tp53 mutations to the mouse liver, Hill et al. ${ }^{17}$ demonstrated that selective induction of Kras and Tp53 mutations in mature hepatocytes in the setting of liver injury, such as DDC-induced chronic inflammation (3,5diethoxycarbonyl-1,4-dihydrocollidine), could drive rapid progression of $\mathrm{ICC}^{17}$. More importantly, Tp53 has been identified as a key regulator in enabling hepatocytederived ICC in this context. Indeed, Tp53 has been shown to control plasticity in a number of different cellular contexts $^{18}$ and thus Tp53 mutations may facilitate such transdifferentiation events that are implicated in hepatocyte-derived ICC pathogenesis ${ }^{19}$.

\section{Tgfbr2}

Another study reported that Tgfbr2 (TGF- $\beta$ receptor II) restricts hepatocyte-derived ICC ${ }^{20}$. TGF- $\beta$ pathway is closely related to the development of hepatic fibrosis both in mice and patients ${ }^{21}$. It is noteworthy that recent exon sequencing revealed a high frequency of mutations in Smad4, a key downstream mediator of TGF- $\beta$ signals, in human cholangiocarcinoma ${ }^{22}$. Most recently, a new study reveals that hepatocyte-specific deletion of Tgfbr2 and PTEN mediated by AAV8-TBG-Cre promoted hepatocyte-derived ICC formation and reduced survival of mice ${ }^{20}$. Mechanistically, deletion of Tgfbr2 promotes the proliferation of cholangiocyte rather than hepatocytes, suggesting the pivotal role of epithelial Tgfbr2 in restricting cholangiocyte proliferation ${ }^{20}$. Although targeting TGF- $\beta$ may be clinically effective for liver fibrosis, this approach may increase the risk of ICC, which needs to be paid enough attention in clinic.

\section{c-Myc}

The recent study of Hill et al. ${ }^{23}$ indicates $\mathrm{c}-\mathrm{Myc}$ is required for hepatocyte-derived ICC in AKT/Fbxw7 $\triangle \mathrm{F}$ mice. The ubiquitin ligase F-box and WD repeat domaincontaining 7 (FBXW7) plays an anti-cancer role in many cancers, such as HCC, colorectal cancer and gastric can$\mathrm{cer}^{24}$. It can lead to the degradation of several oncogenes, such as c-MYC and YAP ${ }^{25}$. Wang et al. ${ }^{23}$ generated a ICC mouse model by co-expression of Fbxw $7 \Delta \mathrm{F}$ (a dominant negative form of Fbxw7) and AKT plasmids in mice livers.
Using lineage tracing technology, they confirmed that ICC lesions induced by $\mathrm{AKT} / \mathrm{Fbxw} 7 \Delta \mathrm{F}$ derived from hepatocytes. Interestingly, selected deletion of $\mathrm{c}-\mathrm{Myc}$, as for the downstream targets of FBXW7, completely suppresses hepatocyte-derived ICC formation in AKT/Fbxw7 $\Delta \mathrm{F}$ mice $^{23}$. Furthermore, in human ICC specimens, the expression level of Fbxw7 was negatively correlated with the transcription activity of $\mathrm{c}-\mathrm{myc}^{34}$. Therefore, c-Myc could serve as a therapeutic target for ICC treatment, especially with respect to patients with low FBXW7 expression.

\section{Ras/MAPK cascade}

Ras/MAPK cascade may influence the formation of hepatocyte-derived ICC by promoting cell proliferation and regulating tumor microenvironment ${ }^{26}$. Previous studies have shown that Ras/MAPK pathway is significantly activated in human $\mathrm{ICC}^{27}$. In a recent study, Wang et al. generated a hepatocyte-derived ICC mouse model by hydrodynamic tail vein injection of AKT and YapS127A plasmids in mice livers ${ }^{26}$. They found that inhibition of Ras/MAPK cascade significantly delayed the progression of AKT/YapS127A-induced ICC. On the one hand, Ras/ MAPK cascade can significantly promote the proliferation of cholangiocarcinoma cells. On the other hand, this cascade can recruit activated hepatic stellate cells (AHSC) and create hypoxic microenvironment in tumor tissues, which is key features of human ICC $^{28}$. Because MEK is a key player in Ras/MAPK pathway, MEK inhibitors may be a therapeutic option for ICC in future clinical trials.

\section{LTRR signaling}

The recent study of Scarzello et al. ${ }^{29}$ indicates LT $\beta R$ signaling accelerates formation of hepatocyte-derived ICC in AKT/ $\beta$-catenin and AKT/NICD mouse models. LT $\beta R$ is a member of the tumor necrosis factor (TNF) superfamily of receptors ${ }^{30}$ and implicated in the initiation of liver cancer ${ }^{31}$. AKT/CAT-induced tumors display multiple pathological features, including lipogenic hepatic foci, HB/HCC-like nodules and ICC-like lesions ${ }^{32}$, among which the first two types of pathological features are most common, while ICC-like lesions are relatively rare. However, when using LT $\beta R$ agonists, more ICC-like tumors were observed in $A K T / \beta$-catenin mouse model, suggesting LT $\beta R$ signaling skews AKT/ $\beta$-catenin pathology towards a more ICC-like phenotype ${ }^{29}$. In addition, a role for LT $\beta R$ signaling in promoting the progression of ICC was further confirmed using AKT/NICD-initiated ICC model. In preclinical and clinical research study of liver cancer, combination therapies are being widely explored and are attracting more attention increasingly. Immune agents blocking the activity of LT $\beta R$ in combination with other drugs, such as Akt or $\beta$-catenin pathway inhibitor, may achieve better therapeutic effect in ICC. 
Tumor microenvironment that commits ICC formation

The so-called tumor microenvironment has been recognized as an important regulator in the initiation, development and treatment of various cancers. Recently, it has been found that necroptosis-associated hepatic cytokine microenvironment facilitates formation of $\mathrm{ICC}^{33}$. Tumor microenvironment is a complex environment for the survival and development of cancer cells, which mainly consists of cellular and non-cellular components ${ }^{34}$. Both components play a supporting role in the growth of tumors ${ }^{35}$. Very recently, it has been found that the microenvironment of cancer cells (especially the special form of cell death occurring in this environment) has a decisive influence on whether HCC or ICC occurs ${ }^{33}$. In necrotic apoptosis, a large number of cytokines are secreted from immune cells that are activated by damageassociated molecular patterns (DAMPs), which released from necroptosically dying hepatocytes ${ }^{36}$. While in apoptosis, vesicles are cleared by the immune system and there is no large amount of cytokine production in microenvironment ${ }^{37}$. Researchers found that hepatocytes with aberrantly activated oncogenes, if the cell death in their environment is caused by apoptosis, will give rise to $\mathrm{HCC}$; on the other hand, if the cell death is caused by necroptosis, it will lead to ICC. These results were further validated in mouse models and human tissue samples ${ }^{33}$. Most importantly, the microenvironment formed by different apoptotic pathways had a great influence on two epigenetic regulators (Tbx3 and Prdm5), which are the key regulator in determining lineage commitment in liver cancer $^{38}$. Interestingly, simultaneous Prdm5 overexpression and Tbx3 knockdown resulted in the development of ICC; however, Tbx3 overexpression combined with Prdm5 knockdown lead to the development of $\mathrm{HCC}^{33}$. There is evidence that inflammatory cytokines and infiltrated immune cells may play an important role in the formation of ICC because they may connect the bridge between the oncogenic driver genes and hepatic death. Seehawer et al. ${ }^{33}$ found that some specific cytokines (e.g., Ccl4, Ccl8, Osm, Ccl6, Cxcl13, Pf4, and Aimp1) are secreted by immune cells, which are activated by DAMPs released from necroptosically dying hepatocytes ${ }^{33}$. These specific cytokines may act on hepatocytes together with aberrantly activated oncogenes and further led to ICC. However, they also found that the infiltrated immune cells (e.g., T cells, monocytes and (neutrophilic) granulocytes as well as B cells and antigen-presenting cells) were not obvious in the necroptosic microenvironment, suggesting the limited role of infiltrated immune cells in ICC formation $^{33}$. In the future, in addition to the types of cancer, we should also study whether the tumor microenvironment directly affects the choice of treatments. In the course of chemoembolization therapy for patients with $\mathrm{HCC}$, we found that some primary HCC could transform into ICC. The possible mechanism may lie in the necroptosis-associated hepatic cytokine microenvironment caused by chemoembolization therapy, which may promote a phenotypic transition from HCC to ICC. This may be one of the important reasons for drug resistance in patients with liver cancer.

\section{Regulatory molecules and tumor microenvironment that may commit cHCC-ICC formation}

cHCC-ICC is described as demonstrating histologic features of both hepatocellular and biliary epithelial differentiation. It is a rare primary liver malignancy, accounting for $1 \%$ to $14.2 \%$ of cases $^{39}$. The diagnosis of cHCC-ICC is usually made at pathologic evaluation after either resection or transplantation and it is practically impossible to achieve an accurate, pre-operative diagnosis of cHCC-ICC with tumor markers or abdominal imaging $^{40}$. Accordingly, the actual incidence may be higher due to frequent difficulty in accurate pathological assessment. Although little is known clinically about this type of malignancy, the data available indicate that it is aggressive and likely signify a unique subset of PLCs, which merits clinical distinction ${ }^{41}$. Recent lineage tracing experiments in mice have demonstrated that some subtypes of liver cancer, such as HCC and ICC, are derived from mature hepatocytes rather than from liver stem/ progenitor cells ${ }^{7}$. Moreover, by analyzing systematic mutations, somatic copy number variations, and clonal analyses of human cHCC-ICC tissues, Moeini et al. ${ }^{42}$ and Wang et al. $^{43}$ demonstrated that HCC and ICC components share a common cell of origin.

Consistently, our previous studies have shown that cHCC-ICC may originate from hepatocytes in AKT/ CAT model. AKT/CAT-initiated tumors display multiple pathological characteristics, including lipogenic hepatic foci, HCC, ICC and CHCC-ICC. We found the moribund livers (5\%) following 2 months of AKT and CAT injection displayed a pathological characteristics of cHCC-ICC containing both HCC and ICC two components (Fig. $3 \mathrm{a}-\mathrm{c}$ ). Some scattered HA-tag strongly positive hepatocytes were detected in AKT/CAT -injected livers after 7 days (Fig. 3d, e). Interestingly enough, after 90 days, IHC results showed that HA-tag protein was also expressed in both HCC and ICC two components of cHCC-ICC tumor tissues (Fig. 3f), indicating that $\mathrm{HCC}$ and ICC components might originate from these HA-tag positive hepatocytes.

Therefore, liver tumors originated from mature hepatocytes consist of a wide spectrum of phenotypes from $\mathrm{HCC}$ to $\mathrm{CC}$, possibly encompassing $\mathrm{CHCC}-\mathrm{ICC}^{4}$. Based on the latest research, mechanism underlying hepatocytederived cHCC-ICC formation can be summarized as the following aspects (Fig. 4). 

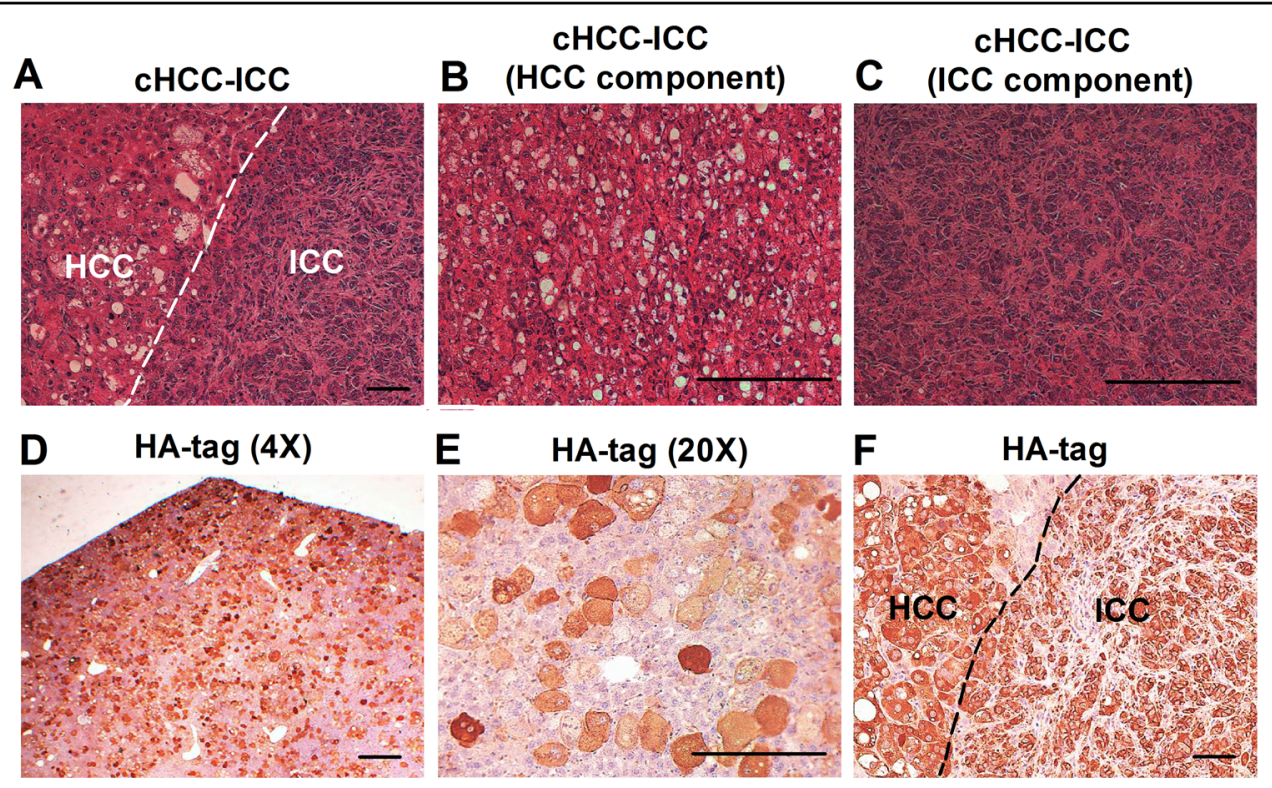

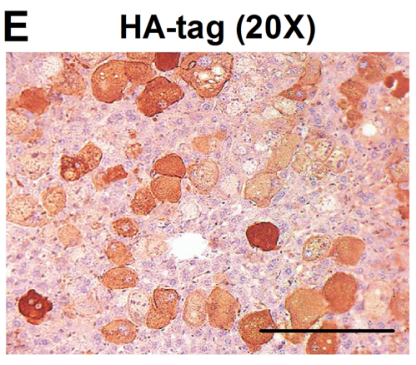

7 days

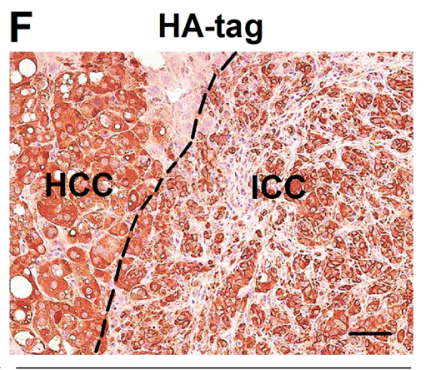

90 days

Fig. 3 HCC and ICC components of CHCC-ICC could both originate from hepatocytes. a-c The moribund livers (5\%) following 2 months of AKT and CAT injection displayed a pathological characteristics of CHCC-ICC containing both HCC and ICC two components. d, e IHC showed that some scattered HA-tag strongly positive hepatocytes were detected in AKT/CAT -injected livers after 7 days. $\mathbf{f} \| \mathrm{HC}$ showed that HA-tag protein was also expressed in both HCC and ICC two components of CHCC-ICC tumor tissues after 90 days. HCC: hepatocellular carcinoma; ICC: intrahepatic cholangiocarcinoma; CHCC-ICC: combined hepatocellular carcinoma and intrahepatic cholangiocarcinoma; IHC: immunohistochemical staining (scale bars, $50 \mu \mathrm{m})$.

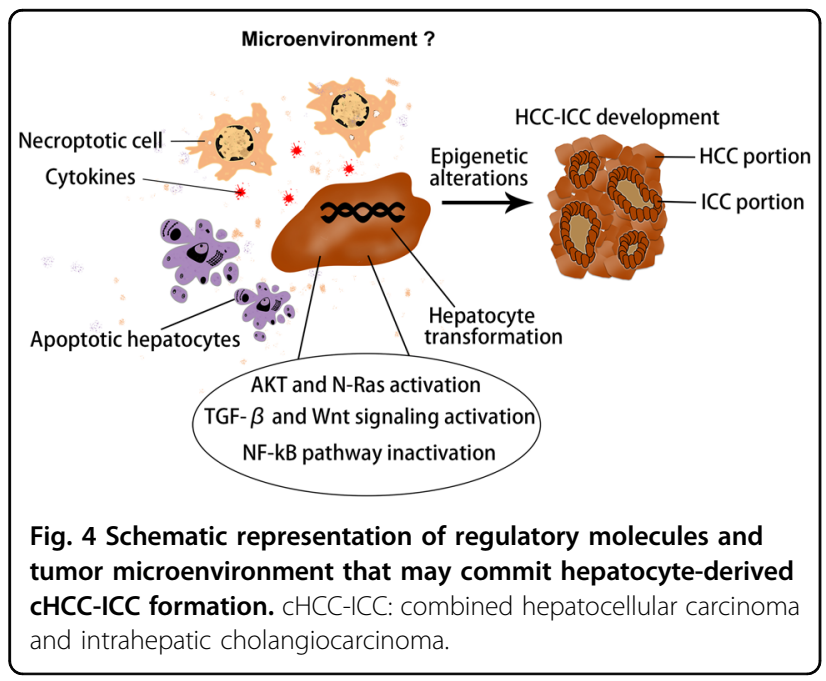

TGF- $\beta$, Wnt, and Notch signaling

TGF- $\beta$, Wnt $/ \beta$-catenin and Notch signalings were identified as the major signaling activated in human cHCC-ICC specimens ${ }^{41}$. Indeed, by using a genome-wide transcriptional analysis, Coulouarn et al.'s ${ }^{44}$ study showed that cHCC-ICC exhibited a gene signature characteristic of the activation of the $\mathrm{Wnt} / \beta$-catenin pathway, which is closely related to the development of bile duct morphology. Interestingly, TGF- $\beta$ signaling pathway has been reported to be activated in cHCC-ICC and could be attributed to the presence of the tumoral fibrous stroma with a cholangiocarcinoma-like gene expression trait ${ }^{45}$. Such results are in accord with a previous study published in Nature, suggesting that TGF- $\beta$ signaling enhances the formation of the biliary system from hepatocytes through a transdifferentiation mechanism ${ }^{46}$. Therefore, TGF- $\beta$ and $\mathrm{Wnt} / \beta$-catenin pathway may be involved in the formation of ICC components in cHCC-ICC. In addition, mutations of TERT promoter and TP53, as well as substantial intratumoral heterogeneity, often appear in cHCC-ICC $^{41}$. Consistently, our previous studies have shown that TGF- $\beta$ and Notch signalings were activated in human cHCC-ICC, especially in the ICC components (Fig. 5).

\section{NF-kB pathway}

The function of NF- $\mathrm{KB}$ in liver cancer is still contradictory. Some studies have shown that the NF- $\mathrm{kB}$ pathway promotes inflammation-related cancer ${ }^{47}$, whereas inhibition of NF- $\mathrm{kB}$ activity in hepatocytes promotes the spontaneous formation of $\mathrm{HCC}$, indicating that the NF- $\mathrm{kB}$ pathway function as tumor suppressor in hepatocytes ${ }^{48}$. A recent study, the first to analyze the role of NF-kB pathway in the progression of $\mathrm{CHCC}-\mathrm{ICC}$, indicates that block of NF-kB signaling skews c-Myc-driven HCC pathology 

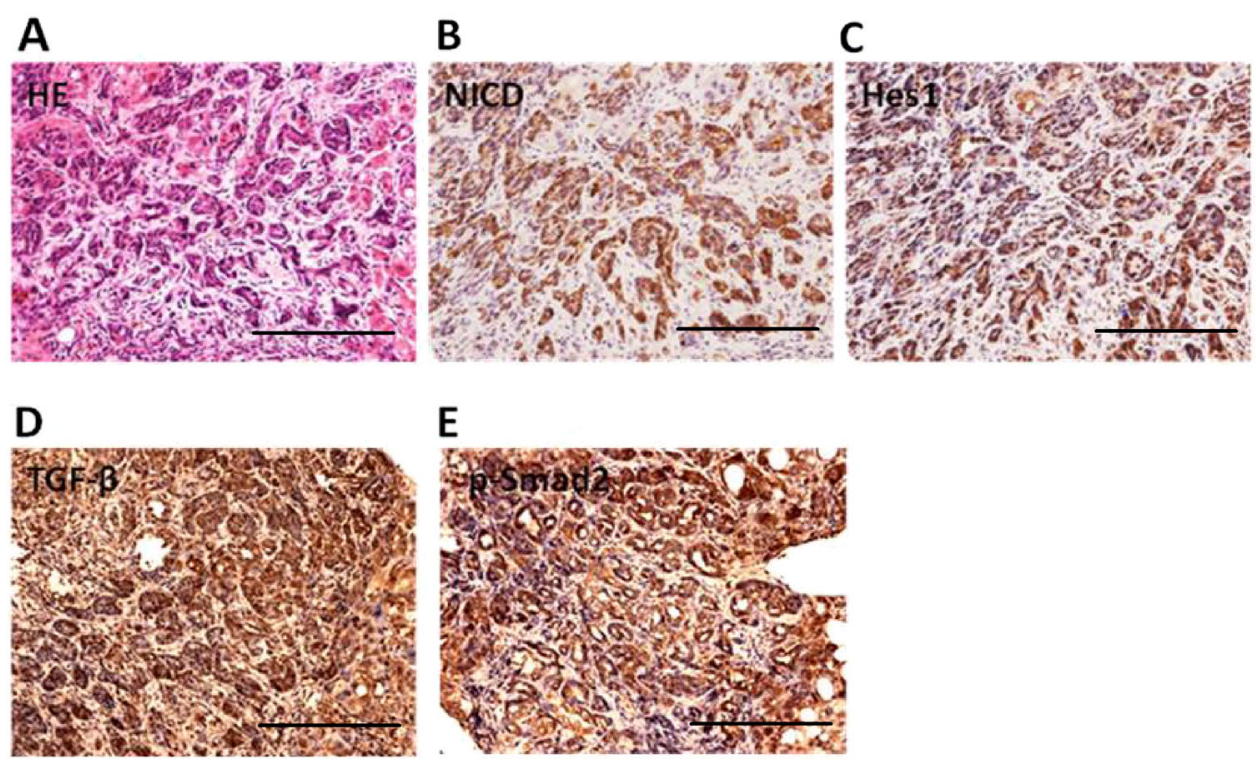

Fig. 5 IHC showed that TGF- $\beta$ and Notch signalings were activated in the ICC components of human cHCC-ICC. ICC: intrahepatic cholangiocarcinoma; CHCC-ICC: combined hepatocellular carcinoma and intrahepatic cholangiocarcinoma; IHC: immunohistochemical staining (scale bars, $50 \mu \mathrm{m})$.

towards a more cHCC-ICC-like phenotype ${ }^{49}$. It was well known that the tumor phenotype induced by c-Myc often manifests as $\mathrm{HB}^{50}$, however, ICC has not been reported previously. Importantly, after inhibition of NF-kB pathway, an additional tumor component resembling ICC was observed in this model, which was accompanied by MAPK activation, reflecting previous reports on the critical role of NF-kB pathway in cholangiocarcinoma ${ }^{51}$. Accumulating evidence suggests that liver cancer phenotype can be influenced by sequential oncogenic dysregulation and the inflammatory milieu ${ }^{29}$. Given NF- $\mathrm{kB}$ deletion led to an increase in infiltrating inflammatory cells $^{52}$, it is reasonable that the chronic inflammatory environment caused by NF- $\mathrm{kB}$ ablation may modulate the phenotypic transition in this model.

\section{AKT and N-Ras (N-Ras-V12, a persistently active form of N-Ras)}

Activation of AKT and Ras pathways is often implicated in hepatocarcinogenesis. A previous study showed that overexpression of AKT and N-Ras in the mouse liver (AKT/Ras) by way of hydrodynamic gene transfer can accelerate both HCC and ICC development (i.e., cHCCICC), with ICC lesions accounting for about $10 \%$ of the total lesion $\operatorname{area}^{53}$. Mechanistically, mTORC1, FOXM1/ SKP2, and c-Myc signaling cascades were found to be involved in the mediating AKT/N-Ras-induced hepatocarcinogenesis $^{54}$. In addition, N-Ras-V12 oncogene was delivered to the livers of p19Arf-null or heterozygous mice to elicit tumor formation. The results showed that the tumor pathological type of this model was cHCC-ICC, further suggesting a key role of N-Ras-V12 in the development of $\mathrm{CHCC}-\mathrm{ICC}^{55}$.

\section{Tumor microenvironment that may commit CHCC-ICC formation}

According to our knowledge, there is no report on the tumor microenvironment of $\mathrm{cHCC}-\mathrm{ICC}$ so far. As mentioned earlier, necroptosis-associated hepatic cytokine microenvironment facilitates formation of hepatocytederived ICC, whereas apoptosis-associated hepatic cytokine microenvironment promotes formation of hepatocyte-HCC. Based on this, we speculate that hepatocytes with aberrantly activated oncogenes, if the cell death in their environment is jointly caused by necroptosis and apoptosis, will give rise to cHCC-ICC (Fig. 4). However, this viewpoint needs to be verified by experiments in the future.

\section{Regulatory molecules and tumor microenvironment that commit HCC formation}

Until recently, some well-established lineage-tracing mouse experiments have further demonstrated that HCC originates from mature hepatocytes rather than LPCs, hepatic stellate cells (HSCs) and biliary compartment both in genotoxic and genetic models ${ }^{7}$. In order to study the molecular mechanism of hepatocyte-derived HCC formation, various primary HCC mouse models were established (Table 1). For instance, mouse HCC induced by $\mathrm{CCl} 4$, diethylnitrosamine (DEN), or aristolochic acid 
Table 1 The various mouse models of liver cancer.

\begin{tabular}{|c|c|c|c|c|}
\hline Genes & Tumor type & Mouse strains & Latency & Reference \\
\hline \multicolumn{5}{|c|}{ Genetically engineered mouse models for liver cancer } \\
\hline AAT & $\mathrm{HCC}$ & $\begin{array}{l}\text { Transgenic mice using human alpha } \\
\text { 1-antitrypsin } \mathrm{M} \text { and Z genomic clones }\end{array}$ & 52-90 weeks & Geller et al..$^{60}$ \\
\hline $\mathrm{NEMO}^{-1-}$ & $\mathrm{HCC}$ & NEMO ${ }^{\Delta \text { hepa }}$ mice & 52 weeks & Beraza et al. ${ }^{61}$ \\
\hline PTEN $^{-/-}$ & $\mathrm{HCC}$ & PTEN-deficient mice & 42-44 weeks & Watanabe et al. ${ }^{62}$ \\
\hline $\mathrm{PTEN}^{-/-}+\mathrm{GRP}^{-1-}$ & CHCC-ICC & $\begin{array}{l}\text { PTEN and GRP94 two liver-specific } \\
\text { knockout mouse }\end{array}$ & 25 weeks & Chen et al. ${ }^{63}$ \\
\hline HCV core & $\mathrm{HCC}$ & Transgenic for the HCV core gene & 80-105 weeks & Moriya et al. ${ }^{64}$ \\
\hline $\mathrm{TAK}^{-/-}$ & $\mathrm{HCC}$ & Tak $1^{\Delta \text { hepa }}$ mice & 39 weeks & Inokuchi et al. ${ }^{65}$ \\
\hline $\mathrm{HBx}$ & $\mathrm{HCC}$ & $\begin{array}{l}\text { Transgenic mice expressed HBV-encoded } \\
\text { gene products }\end{array}$ & 52-104 weeks & Chisari et al. ${ }^{66}$ \\
\hline $\mathrm{KRAS}^{\mathrm{G} 12 \mathrm{D}}+\mathrm{HBx}$ & $\mathrm{HCC}$ & $\operatorname{Kras}(\mathrm{G} 12 \mathrm{D})$ and $\mathrm{HBx}$ double transgenic mice & 34 weeks & Ye et al. ${ }^{67}$ \\
\hline c-myc & $H B$ & c-myc single transgenic mice & 65-90 weeks & $\begin{array}{l}\text { Thorgeirsson } \\
\text { et al. }{ }^{68}\end{array}$ \\
\hline$c-m y c+E G F$ & $\mathrm{HCC}$ & $\begin{array}{l}\text { Autocrine growth factor lgEGF and c-myc } \\
\text { single transgenic mice }\end{array}$ & $12-18$ weeks & Tönjes et al. ${ }^{69}$ \\
\hline$c-m y c+E 2 F 1$ & $\mathrm{HCC}$ & c-Myc/E2F1 transgenic mouse & 26-39 weeks & Calvisi et al. ${ }^{70}$ \\
\hline $\mathrm{P} 53^{-1-}+\mathrm{c}-\mathrm{myc}$ & $\mathrm{HCC}$ & c-Myc/p53KO mice & 21 weeks & Klocke et al. ${ }^{71}$ \\
\hline $\mathrm{P}^{2} 3^{-1-}$ & $\mathrm{HCC}$ & P55 $3^{\Delta \text { hepa }}$ mice & 60 weeks & Katz et al. ${ }^{72}$ \\
\hline EGF & $\mathrm{HCC}$ & $\begin{array}{l}\text { Autocrine growth factor lgEGF } \\
\text { transgenic mice }\end{array}$ & 24-36 weeks & Tönjes et al. ${ }^{69}$ \\
\hline SV40 T-antigen & $\mathrm{HCC}$ & Mice expressing SV 40 early sequences & 20 weeks & Lou et al. ${ }^{73}$ \\
\hline E2F-1 & $\mathrm{HCC}$ & E2f1 transgenic mice & 52 weeks & Lee et $\mathrm{al}^{74}$ \\
\hline $\mathrm{APC}^{-1-}$ & $\mathrm{HCC}$ & $\mathrm{APC}^{\Delta \text { hepa }}$ mice & 38 weeks & Colnot et al. ${ }^{75}$ \\
\hline TGF- a & $\mathrm{HCC}$ & TGF-alpha transgenic mice & $>52$ weeks & Lee et al. $^{76}$ \\
\hline$\beta$-catenin(Dex3) + HRASG12V & $\mathrm{HCC}$ & $\begin{array}{l}\text { Mouse strain containing a mutant beta- } \\
\text { catenin allele of which exon } 3 \text { was } \\
\text { sandwiched by loxP sequences [Catnb(lox } \\
(\text { ex3))] }\end{array}$ & 8 weeks & Harada et al. ${ }^{77}$ \\
\hline \multicolumn{5}{|c|}{ Application of the hydrodynamic transfection methodology to induce liver cancer } \\
\hline myr-AKT & $\mathrm{HCC}$ & C57BL/6J, FVB/N & 6 months & Calvisi et al. ${ }^{78}$ \\
\hline myr-AKT and DN90-b-catenin & $\mathrm{HCC}$ & C57BL/6J, FVB/N & 1 months & Calvisi DF et al. ${ }^{79}$ \\
\hline myr-AKT and NRasV12 & CHCC-ICC & C57BL/6J, FVB/N & 1 months & Ho et al. $^{54}$ \\
\hline myr-AKT and NICD & ICC & C57BL/6J, FVB/N & 3 weeks & Fan et al. ${ }^{6}$ \\
\hline c-Met and DN90-b-catenin & $\mathrm{HCC}$ & C57BL/6J, FVB/N & 3 months & Tward et al. ${ }^{80}$ \\
\hline NRasV12 and DN90-b-catenin & $\mathrm{HCC}$ & C57BL/6J, FVB/N & 3 months & Lee et $a .^{81}$ \\
\hline NEMO (IKKү) KO + c-Myc & $\mathrm{CHCC}-\mathrm{ICC}$ & C57BL/6J, FVB/N & 45 days & He et al. $^{49}$ \\
\hline Myc and human NRASG12V & $\mathrm{HCC}$ & p19Arf ${ }^{-/-}$ & 4 weeks & Seehawer et al. ${ }^{33}$ \\
\hline mouse Myc and Akt1 & $\mathrm{HCC}$ & p19Arf ${ }^{-/-}$ & 4 weeks & Seehawer et al. ${ }^{33}$ \\
\hline FAK and DN90-b-catenin & $\mathrm{HCC}$ & 57BL/6J, FVB/N & 24 weeks & Shang et al. ${ }^{82}$ \\
\hline myr-AKT and c-Myc & $\mathrm{HCC}$ & 57BL/6J, FVB/N & 8 weeks & Yamamoto et al. ${ }^{4}$ \\
\hline
\end{tabular}


Table 1 continued

\begin{tabular}{|c|c|c|c|c|}
\hline Genes & Tumor type & Mouse strains & Latency & Reference \\
\hline myr-AKT/c-Myc/YAP & $\mathrm{HCC}$ & $57 \mathrm{BL} / 6 \mathrm{~J}, \mathrm{FVB} / \mathrm{N}$ & 3 weeks & Yamamoto et al. ${ }^{4}$ \\
\hline myr-AKT and YAP & ICC & 57BL/6J, FVB/N & 6 weeks & Yamamoto et al. ${ }^{4}$ \\
\hline c-Myc and YAP & $\mathrm{HB}$ & 57BL/6J, FVB/N & 16 weeks & Yamamoto et al. ${ }^{4}$ \\
\hline NICD1 & ICC & $57 \mathrm{BL} / 6 \mathrm{~J}, \mathrm{FVB} / \mathrm{N}$ & 5 months & Fan et al. ${ }^{6}$ \\
\hline HRasV12 and shP53 & $\begin{array}{l}\text { Undifferentiated } \\
\text { liver tumors }\end{array}$ & 57BL/6J, FVB/N & 1 week & Ju et al. ${ }^{83}$ \\
\hline NRasV12 & CHCC-ICC & Ink4A/Arf ${ }^{-1-}$ & 7 weeks & Carlson et al. ${ }^{55}$ \\
\hline myr-AKT and Spry2Y55F & $\mathrm{HCC}$ & $57 \mathrm{BL} / 6 \mathrm{~J}, \mathrm{FVB} / \mathrm{N}$ & 4 months & Wang et al..$^{84}$ \\
\hline c-Myc and shp53 & $\mathrm{HCC}$ & $57 \mathrm{BL} / 6 \mathrm{~J}, \mathrm{FVB} / \mathrm{N}$ & 7 months & Ju et al. ${ }^{85}$ \\
\hline AKT/Fbxw7 $\triangle F$ & ICC & $57 \mathrm{BL} / 6 \mathrm{~J}, \mathrm{FVB} / \mathrm{N}$ & 10 weeks & Wang et al..$^{23}$ \\
\hline Nras-FAH and shP53 & $\mathrm{HCC}$ & $\mathrm{Fah}^{-1-}$ & 10 weeks & $\begin{array}{l}\text { Wangensteen } \\
\text { et al. }^{86}\end{array}$ \\
\hline Bmi1 and NRasV12 & $\mathrm{HCC}$ & $57 \mathrm{BL} / 6 \mathrm{~J}, \mathrm{FVB} / \mathrm{N}$ & 6 months & Xu et al. ${ }^{87}$ \\
\hline \multicolumn{5}{|c|}{ Application of the chemical carcinogens to induce liver cancer } \\
\hline Diethylnitrosamine (DEN) & $\mathrm{HCC}$ & $57 \mathrm{BL} / 6 \mathrm{~J}, \mathrm{FVB} / \mathrm{N}$ & 14 months & Ngo et al. ${ }^{57}$ \\
\hline Aristolochic acid & $\mathrm{HCC}, \mathrm{CHCC}-\mathrm{ICC}$ & 57BL/6J, FVB/N & $\begin{array}{l}\text { In a dose- } \\
\text { dependent manner }\end{array}$ & Lu et al. ${ }^{58}$ \\
\hline
\end{tabular}

was often accompanied with reactivation of a variety of fetal liver genes, such as Gpc3, Afp, Slpi, Spink3, and $\mathrm{Abcd} 2^{56-58}$. Moreover, various transgenic mouse models of HCC have been successfully generated by overexpression of oncogenes such as AKT, Myc, Bmil, c-Met, Tgfa, E2F1, Ccnd1, Spry2Y55F, and HRASG12V, or genes that encode viral proteins, such as HbsAg, HBX, and SV40 $\mathrm{T}$-Ag (Table 1$)^{59-77}$. However, these transgenic mouse models have several limitations, such as high costs, time consuming and requiring high professional knowledge and skills. Hydrodynamic gene delivery is a new method that combines with the SB mediated somatic integration for long-term gene expression in mouse hepatocytes, which has been used in developing novel murine models

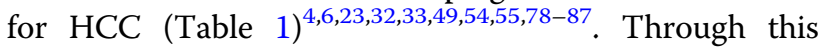
technique, Che et al ${ }^{88}$ reveals a novel crosstalk between aberrant lipogenesis and cholesterol biosynthesis pathways in the progression of HCC. Shang et al. ${ }^{89}$ demonstrated that co-overexpression of focal adhesion kinase (FAK) and $\beta$-Catenin leads to HCC formation. Therefore, hydrodynamic transfection is a reliable method to induce liver tumor and can be used to study the role of genes with unknown functions in hepatocarcinogenesis.

Consistently, our previous studies have shown that HCC may originate from hepatocytes in AKT mouse model. AKT-initiated tumors were characterized by lipid rich droplets and high proliferation (Fig. 6c, d). Some scattered hepatocytes with strongly positive HA-tag were



Fig. 6 HCC could originate from hepatocytes. a $I \mathrm{HC}$ showed that some scattered HA-tag strongly positive hepatocytes were detected in AKT-injected livers after 7 days. b $\mathrm{IHC}$ results showed that HA-tag protein was also expressed in HCC tumor tissues after 6 months. $\mathbf{c ~ H E}$ staining showed that AKT-initiated tumors were characterized by lipid rich droplets. $\mathbf{d} I \mathrm{HC}$ results showed that PCNA protein was highly expressed in HCC tumor tissues after 6 months. HCC: hepatocellular carcinoma; IHC: immunohistochemical staining; HE: hematoxylin-eosin staining (scale bars, $50 \mu \mathrm{m}$ ). 




detected in AKT-injected livers after 7 days (Fig. 6a). IHC results showed that HA-tag protein was also expressed in HCC tumor tissues after 6 months (Fig. 6b), indicating that HCC might originate from these HA-tag positive hepatocytes. Based on the latest research, mechanism underlying hepatocyte-derived HCC formation can be summarized as the following aspects (Fig. 7).

\section{Hepatocarcinogenesis due to the interaction of multiple genes}

The occurrence of HCC is a complex process accompanied by the activation of multiple signaling pathways, which plays a synergistic role in the process of tumorigenesis ${ }^{90}$. Numerous studies have confirmed that PI3K/ AKT/mTOR pathway and $\mathrm{Wnt} / \beta$-catenin pathways play an important role in the development of $\mathrm{HCC}^{91}$. For instance, hydrodynamical codelivery of activated forms of AKT (pT3-EF1 $\alpha$-HA-myr-AKT) and $\beta$-catenin (pT3EF1 $\alpha-\Delta 90 \beta$-catenin, CAT) oncogenes into mouse livers using the SB transposon system efficiently and rapidly induces primary hepatic tumors. AKT/CAT-initiated tumors display multiple pathological characteristics, including early lipogenic hepatic foci and subsequent $\mathrm{HB}$ / HCC-like nodules, which is rich in lipids ${ }^{29}$. Importantly, this provides a good animal model for the study of steatosis-related liver cancer. In addition, the activated form of AKT was found to cooperate with activated Myc, Yap, NRasV12 or Spry2Y55F pathways to induce HCC formation in the mouse ${ }^{84}$. Hydrodynamical codelivery of the activated mutant of $\beta$-catenin and $\mathrm{c}-\mathrm{MET}^{1}$ or NRasV12 ${ }^{81}$ into mouse livers can also efficiently induce HCC over a short latency. Using the same method, Li et al. ${ }^{92}$ reported that the introduction of YAPS ${ }^{127 \mathrm{~A}}$ and PIK3CA ${ }^{\text {H1047R }}$ (a constitutively active mutant of PI3K) induces liver cancers with many pathological features.
Fan et al. $^{93}$ found that Bmil is required for AKT/Ras -induced HCC development. Altogether, these results reflect the complex interaction of different oncogenes in hepatocyte-derived HCC formation.

\section{A single gene sufficient for hepatocarcinogenesis}

Activated PI3K/Akt/mTOR pathway is closely related to poor differentiation, early recurrence and poor prognosis of $\mathrm{HCC}^{94}$. Four weeks after hydrodynamical delivery of AKT plasmids, the livers are pale and greasy. Microscopically, hepatocytes were abundant with cytoplasmic lipid and characterized by the intermingled small ductular structures ${ }^{95}$. After 22-32 weeks of transfection, all AKT mice developed lethal liver cancer. In general, the livers of AKT mice were pale and enlarged. There were many tumor nodules on the surface. Microscopically, these tumor cells were characterized by increased cell volume and transparent cytoplasm due to fat accumulation ${ }^{95}$. This suggests that overexpression of AKT alone is sufficient to form liver cancer. For another example, MYC oncogene has been implicated in human liver cancer ${ }^{96}$. It was reported that MYC was over expressed in over $70 \%$ of viral or alcohol-related human $\mathrm{HCC}^{96}$. Hydrodynamic transfection of MYC caused lethal burden of liver cancer by 6-8 weeks post injection. Pathologically, MYC tumors are poorly differentiated and resemble human HBs with cancer stem cells-like properties ${ }^{97}$. All these studies demonstrate that a single gene, such as MYC or AKT, is sufficient for hepatocarcinogenesis, even if not combined with other oncogenes.

\section{Tumor microenvironment that commits HCC formation}

Chronic liver inflammation has been implicated in tumorigenesis. Actually, most HCC develops in an inflammatory environment caused by viral hepatitis and alcoholic or nonalcoholic steatohepatitis ${ }^{98}$. Recent studies have shown that inflammation microenvironment can induce transformation of tumor types. For example, Matter et al. ${ }^{99}$ demonstrated that chronic liver inflammation caused by DDC (3,5-diethoxycarbonyl-1,4-dihydrocollidine) changed AKT/CAT-induced tumors pathology. AKT/CAT-induced tumors were steatotic and contained lipid droplets, whereas lipid content in tumors of AKT/CAT with DDC group was decreased significantly. Pathological types of AKT/CAT-induced liver cancer can be classified into three types: hepatocellular adenoma (HCA), HCC, and HB. In AKT/CAT group, the proportion of HCC was 5-25\%, while in AKT/CAT with DDC group, the proportion of HCC was $5-50 \%$, suggesting that chronic inflammation promotes the phenotypic transition from HCA to HCC. Likewise, chronic inflammation microenvironment induced by DDC can also reduce lipid droplets in AKT-NRAS ${ }^{\mathrm{G} 12 \mathrm{~V}}$ tumors $^{99}$. Altogether, this illustrates that driving oncogenes and 


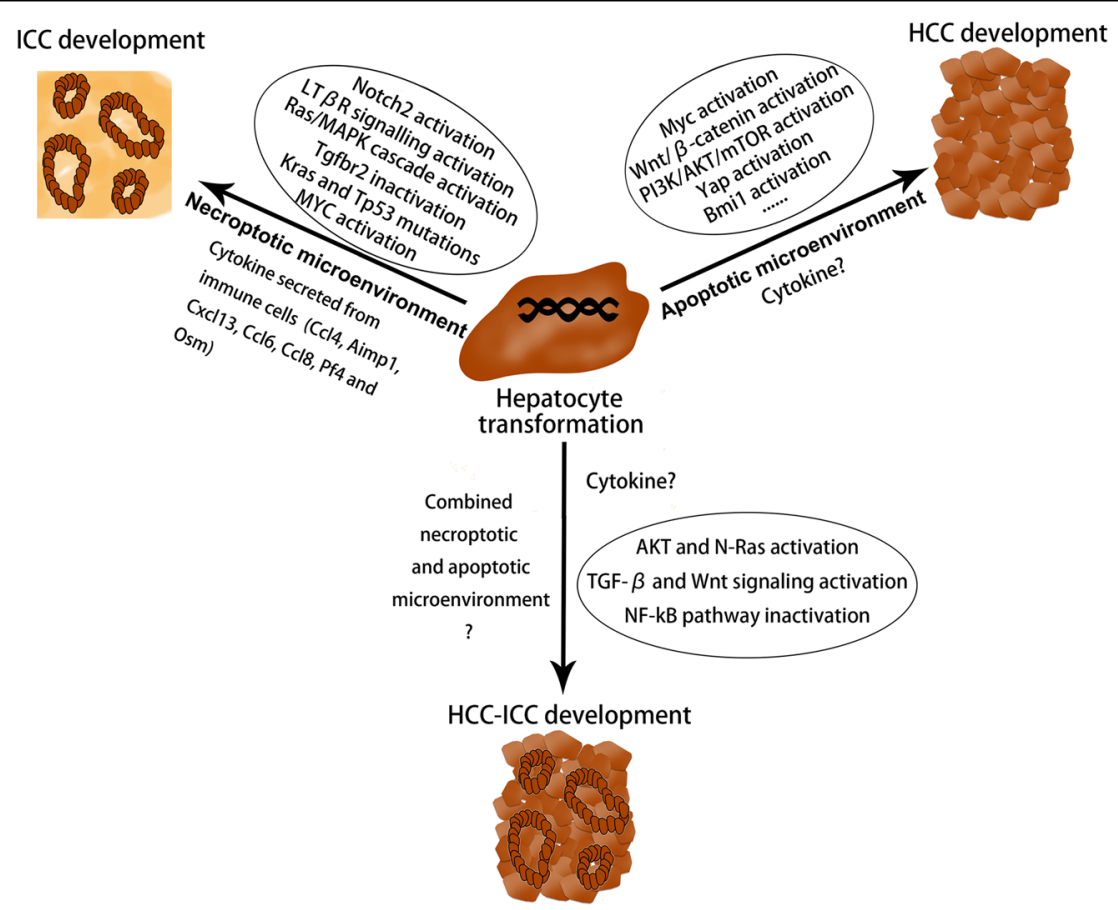

Fig. 8 Oncogenic driver genes and tumor microenvironment determine the type of liver cancer. HCC: hepatocellular carcinoma; ICC: intrahepatic cholangiocarcinoma; CHCC-ICC: combined hepatocellular carcinoma and intrahepatic cholangiocarcinoma.

tumor microenvironment jointly determined the hepatocyte-derived HCC formation.

In summary, this review summarizes the possible mechanism of lineage determination in the development of PLC, including ICC, HCC, and cHCC-ICC (Fig. 8). We put forward the notion that the combined effects of oncogenic driver genes and tumor microenvironment decides the cancer phenotype of hepatocyte-derived mouse liver tumors. PLC always occurs inevitably in a variety of tumor microenvironments, in which different types of cell death such as necrosis, apoptosis or necroptosis occur. It is noteworthy that hepatocytes with aberrantly activated oncogenes will lead to ICC when cell death in their environment is caused by necroptosis with lots of cytokines production. In addition, various intracellular signaling cascades such as Notch2, MYC, Tgfbr2, and Ras/MAPK pathway in hepatocytes mediate the hepatocyte-derived ICC formation (Fig. 2). On the other hand, if the cell death in their environment is caused by apoptosis, hepatocytes with aberrantly activated oncogenes will give rise to HCC. It is well known that some classical cancer-related signalings such as MYC, Yap, Bmil, Wnt/ $\beta$-catenin, and PI3K/AKT/mTOR pathways were implicated in the hepatocyte-derived HCC formation (Fig. 7). cHCC-ICC is a rare primary liver malignancy and the incidence is increasing in the last twenty years, however, its pathogenesis is still poorly understood. Future work is needed to determine whether necroptosis, apoptosis or both occur in the tumor microenvironment that mediate the hepatocyte-derived cHCC-ICC formation (Fig. 4). In conclusion, the possible mechanism of lineage determination in the development of PLC has yet to be delineated. Deciphering the detailed roles of oncogenic driver genes and tumor microenvironment in PLC would certainly pave the way for the development of novel therapies.

\section{Acknowledgements}

The pT3-myr-AKT-HA, pT3-N90-beta-catenin and pT3-EF1a-NICD plasmids were kind gifts from Xin Chen and were obtained from Addgene (Cambridge, MA). pCMV/SB10 was a gift from Perry Hackett and were obtained from Addgene (Cambridge, MA). The authors thank Dr Xin Chen (UCSF, University of California, San Francisco campus) and Dr Perry Hackett (University of Minnesota) for sharing these plasmids. This work was supported by grants from National Natural Science Foundation of China (grant\#81903075, 81670587) and Shanghai Excellent Youth training program (2018YQ62).

\section{Author details}

'Department of General Surgery, The 74th Group Army Hospital, Guangzhou 510220, China. ${ }^{2}$ Department of General Surgery, Tangdu Hospital, Air Force Military Medical University, Xi'an 710032 Shaanxi, China. ${ }^{3}$ Department of Anorectal Surgery, First Affiliated Hospital, Zhengzhou University, Zhengzhou 450052, China. ${ }^{4}$ Department of General Surgery, The 75th Group Army Hospital, Dali 671000, China. ${ }^{5}$ Department of General Surgery, Huashan North Hospital, Fudan University, Shanghai 201907, China. 'Department of Pain Treatment, Tangdu Hospital, Air Force Military Medical University, Xi'an 710032 Shanxi, China. ${ }^{7}$ Department of Dermatology, Dermatology Hospital of Southern Medical University, Guangzhou 510091, China. ${ }^{8}$ Department of General Surgery, Chinese PLA General Hospital, Beijing, China. ${ }^{9}$ Department of Cardiology, The 74th Group Army Hospital, Guangzhou 510318, China.

${ }^{10}$ Departmentof Neurosurgery, First Affiliated Hospital, Zhengzhou University, Zheng zhou 450052, China. "'Department of Anorectal Surgery, The First 
Affiliated Hospital of Zhejiang University, Hangzhou 310003, China.

${ }^{12}$ Department of Infectious Diseases, Xijing Hospital, Air Force Military Medical University, Xi'an 710032 Shaanxi, China. ${ }^{13}$ State key Laboratory of Cancer Biology, National Clinical Research Center for Digestive Diseases and Xijing Hospital of Digestive Diseases, Air Force Military Medical University, Xi'an 710032 Shaanxi, China

\section{Conflict of interest}

The authors declare that they have no conflict of interest.

\section{Publisher's note}

Springer Nature remains neutral with regard to jurisdictional claims in published maps and institutional affiliations.

Received: 17 February 2020 Revised: 10 April 2020 Accepted: 14 April 2020 Published online: 04 May 2020

\section{References}

1. Shang, N. et al. FAK is required for c-Met/beta-catenin-driven hepatocarcinogenesis. Hepatology 61, 214-226 (2015).

2. Connell, L. C., Harding, J. J., Shia, J. \& Abou-Alfa, G. K. Combined intrahepatic cholangiocarcinoma and hepatocellular carcinoma. Chin. Clin. Oncol. 5, 66 (2016).

3. Bergquist, J. R. et al. Mixed hepatocellular and cholangiocarcinoma: a rare tumor with a mix of parent phenotypic characteristics. HPB (Oxf.) 18, 886-892 (2016).

4. Yamamoto, M. et al. Oncogenic determination of a broad spectrum of phenotypes of hepatocyte-derived mouse liver tumors. Am. J. Pathol. 187, 2711-2725 (2017).

5. Lee, J. S. et al. A novel prognostic subtype of human hepatocellular carcinoma derived from hepatic progenitor cells. Nat. Med. 12, 410-416 (2006).

6. Fan, B. et al. Cholangiocarcinomas can originate from hepatocytes in mice. J. Clin. Invest. 122, 2911-2915 (2012).

7. $\mathrm{Mu}, \mathrm{X}$. et al. Hepatocellular carcinoma originates from hepatocytes and not from the progenitor/biliary compartment. J. Clin. Invest. 125, 3891-3903 (2015).

8. Marquardt, J. U., Andersen, J. B. \& Thorgeirsson, S. S. Functional and genetic deconstruction of the cellular origin in liver cancer. Nat. Rev. Cancer 15 653-667 (2015).

9. Nagahama, Y. et al. Contributions of hepatocytes and bile ductular cells in ductular reactions and remodeling of the biliary system after chronic liver injury. Am. J. Pathol. 184, 3001-3012 (2014).

10. Nishikawa, Y. et al. Transdifferentiation of mature rat hepatocytes into bile duct-like cells in vitro. Am. J. Pathol. 166, 1077-1088 (2005).

11. Michalopoulos, G. K., Barua, L. \& Bowen, W. C. Transdifferentiation of rat hepatocytes into biliary cells after bile duct ligation and toxic biliary injury. Hepatology 41, 535-544 (2005).

12. Sekiya, S. \& Suzuki, A. Intrahepatic cholangiocarcinoma can arise from Notchmediated conversion of hepatocytes. J. Clin. Invest. 122, 3914-3918 (2012).

13. Gürlevik, E. et al. Adjuvant gemcitabine therapy improves survival in a locally induced, R0-resectable model of metastatic intrahepatic cholangiocarcinoma. Hepatology 58, 1031-1041 (2013).

14. Wang, J. et al. Notch2 controls hepatocyte-derived cholangiocarcinoma formation in mice. Oncogene 37, 3229-3242 (2018).

15. Zhang, R., Engler, A. \& Taylor, V. Notch: an interactive player in neurogenesis and disease. Cell Tissue Res. 371, 73-89 (2018).

16. Yanger, $\mathrm{K}$. et al. Robust cellular reprogramming occurs spontaneously during liver regeneration. Genes Dev. 27, 719-724 (2013).

17. Hill, M. A. et al. Kras and Tp53 mutations cause cholangiocyte- and hepatocyte-derived cholangiocarcinoma. Cancer Res. 78, 4445-4451 (2018).

18. Jiang, $\mathrm{H}$. et al. Cell cycle and p53 gate the direct conversion of human fibroblasts to dopaminergic neurons. Nat. Commun. 6, 10100 (2015).

19. Llovet, J. M. et al. Hepatocellular carcinoma. Nat. Rev. Dis. Prim. 2, 16018 (2016),

20. $\mathrm{Mu}, \mathrm{X}$. et al. Epithelial transforming growth factor-beta signaling does not contribute to liver fibrosis but protects mice from cholangiocarcinoma. Gastroenterology 150, 720-733 (2016).

21. Bataller, R. \& Brenner, D. A. Liver fibrosis. J. Clin. Invest. 115, 209-218 (2005).
22. Chan-On, W. et al. Exome sequencing identifies distinct mutational patterns in liver fluke-related and non-infection-related bile duct cancers. Nat. Genet. 45 , 1474-1478 (2013)

23. Wang, J. et al. Loss of Fbxw7 synergizes with activated Akt signaling to promote c-Myc dependent cholangiocarcinogenesis. J. Hepatol. 71, 742-752 (2019).

24. Yeh, C. H., Bellon, M. \& Nicot, C. FBXW7: a critical tumor suppressor of human cancers. Mol. Cancer 17, 115 (2018).

25. Tu, K. et al. Fbxw7 is an independent prognostic marker and induces apoptosis and growth arrest by regulating YAP abundance in hepatocellular carcinoma. Mol. Cancer 13, 110 (2014).

26. Zhang, X. F. et al. Expression pattern of cancer-associated fibroblast and its clinical relevance in intrahepatic cholangiocarcinoma. Hum. Pathol. 65, 92-100 (2017).

27. Dong, M. J. et al. Efficacy of MEK inhibition in a K-Ras-driven cholangiocarcinoma preclinical model. Cell Death Dis. 9, 31 (2018).

28. Sha, M. et al. Isolation of cancer-associated fibroblasts and its promotion to the progression of intrahepatic cholangiocarcinoma. Cancer Med. 7, 4665-4677 (2018).

29. Scarzello, A. J. et al. LTbetaR signalling preferentially accelerates oncogenic AKT-initiated liver tumours. Gut 65, 1765-1775 (2016).

30. Ware, C. F. Targeting lymphocyte activation through the lymphotoxin and LIGHT pathways. Immunol. Rev. 223, 186-201 (2008)

31. Haybaeck, J. et al. A lymphotoxin-driven pathway to hepatocellular carcinoma. Cancer Cell 16, 295-308 (2009).

32. Stauffer, J. K. et al. Coactivation of AKT and beta-catenin in mice rapidly induces formation of lipogenic liver tumors. Cancer Res. 71, 2718-2727 (2011).

33. Seehawer, M. et al. Necroptosis microenvironment directs lineage commitment in liver cancer. Nature 562, 69-75 (2018).

34. Lequeux, A. et al. Impact of hypoxic tumor microenvironment and tumor cell plasticity on the expression of immune checkpoints. Cancer Lett. 458, 13-20 (2019).

35. Lin, C. C. \& Korc, M. Designer hydrogels: shedding light on the physical chemistry of the pancreatic cancer microenvironment. Cancer Lett. 436, 22-27 (2018).

36. Ou, L. et al. The mechanisms of graphene-based materials-induced programmed cell death: a review of apoptosis, autophagy, and programmed necrosis. Int. J. Nanomed. 12, 6633-6646 (2017).

37. Elmore, S. Apoptosis: a review of programmed cell death. Toxicol. Pathol. 35, 495-516 (2007).

38. Suzuki, A., Sekiya, S., Buscher, D., Izpisúa Belmonte, J. C. \& Taniguchi, H. Tbx3 controls the fate of hepatic progenitor cells in liver development by suppressing p19ARF expression. Development 135, 1589-1595 (2008).

39. Wang, J., Wang, F. \& Kessinger, A. Outcome of combined hepatocellular and cholangiocarcinoma of the liver. J. Oncol. 8, 2010 (2010).

40. Panjala, C. et al. The diagnostic conundrum and liver transplantation outcome for combined hepatocellular-cholangiocarcinoma. Am. J. Transpl. 10 1263-1267 (2010).

41. Brunt, E. et al. CHCC-CCA: consensus terminology for primary liver carcinomas with both hepatocytic and cholangiocytic differentation. Hepatology $\mathbf{6 8}$ 113-126 (2018).

42. Moeini, A. et al. Mixed hepatocellular cholangiocarcinoma tumors: Cholangiolocellular carcinoma is a distinct molecular entity. J. Hepatol. 66, 952-961 (2017).

43. Wang, A. et al. Whole-exome sequencing reveals the origin and evolution of hepato-cholangiocarcinoma. Nat. Commun. 9, 894 (2018).

44. Coulouarn, $\mathrm{C}$. et al. Combined hepatocellular-cholangiocarcinomas exhibit progenitor features and activation of Wnt and TGFbeta signaling pathways. Carcinogenesis 33, 1791-1796 (2012).

45. Seok, J. Y. et al. A fibrous stromal component in hepatocellular carcinoma reveals a cholangiocarcinoma-like gene expression trait and epithelialmesenchymal transition. Hepatology 55, 1776-1786 (2012).

46. Schaub, J. R. et al. De novo formation of the biliary system by TGFbetamediated hepatocyte transdifferentiation. Nature 557, 247-251 (2018).

47. Pikarsky, E. et al. NF-kappaB functions as a tumour promoter in inflammationassociated cancer. Nature 431, 461-466 (2004).

48. Sakurai, T., Maeda, S., Chang, L. \& Karin, M. Loss of hepatic NF-kappa B activity enhances chemical hepatocarcinogenesis through sustained c-Jun N-terminal kinase 1 activation. Proc. Natl Acad. Sci. USA 103, 10544-10551 (2006). 
49. He, J. et al. Block of NF-kB signaling accelerates MYC-driven hepatocellular carcinogenesis and modifies the tumor phenotype towards combined hepatocellular cholangiocarcinoma. Cancer Lett. 458, 113-122 (2019).

50. Chow, E. K., Fan, L. L., Chen, X. \& Bishop, J. M. Oncogene-specific formation of chemoresistant murine hepatic cancer stem cells. Hepatology 56, 1331-1341 (2012).

51. Wang, C. et al. A systems biology perspective on cholangiocellular carcinoma development: focus on MAPK-signaling and the extracellular environment. J. Hepatol. 50, 1122-1131 (2009).

52. Luedde, T. et al. Deletion of NEMO/KKgamma in liver parenchymal cells causes steatohepatitis and hepatocellular carcinoma. Cancer Cell 11, 119-132 (2007).

53. Li, L. et al. Differential requirement for de novo lipogenesis in cholangiocarcinoma and hepatocellular carcinoma of mice and humans. Hepatology $\mathbf{6 3}$, 1900-1913 (2016).

54. Ho, C. et al. AKT (v-akt murine thymoma viral oncogene homolog 1) and NRas (neuroblastoma ras viral oncogene homolog) coactivation in the mouse liver promotes rapid carcinogenesis by way of mTOR (mammalian target of rapamycin complex 1), FOXM1 (forkhead box M1)/SKP2, and c-Myc pathways. Hepatology 55, 833-845 (2012).

55. Carlson, C. M., Frandsen, J. L., Kirchhof, N., Mclvor, R. S. \& Largaespada, D. A. Somatic integration of an oncogene-harboring Sleeping Beauty transposon models liver tumor development in the mouse. Proc. Natl Acad. Sci. USA 102, 17059-17064 (2005).

56. Chen, $X$. et al. Differential reactivation of fetal/neonatal genes in mouse liver tumors induced in cirrhotic and non-cirrhotic conditions. Cancer Sci. 106, 972-981 (2015).

57. Ngo, H. K. C., Kim, D. H., Cha, Y. N., Na, H. K. \& Surh, Y. J. Nrf2 mutagenic activation drives hepatocarcinogenesis. Cancer Res. 77, 4797-4808 (2017).

58. Lu, Z. N. et al. The mutational features of aristolochic acid-induced mouse and human liver cancers. Hepatology 71, 929-942 (2020).

59. Lee, J. S., Grisham, J. W. \& Thorgeirsson, S. S. Comparative functional genomics for identifying models of human cancer. Carcinogenesis 26, 1013-1020 (2005).

60. Geller, S. A. et al. Hepatocarcinogenesis is the sequel to hepatitis in Z\#2 alpha 1-antitrypsin transgenic mice: histopathological and DNA ploidy studies. Hepatology 19, 389-397 (1994)

61. Beraza, N. et al. Hepatocyte-specific NEMO deletion promotes NK/NKT celland TRAIL-dependent liver damage. J. Exp. Med. 206, 1727-1737 (2009).

62. Watanabe, S. et al. Non-alcoholic steatohepatitis and hepatocellular carcinoma: lessons from hepatocyte-specific phosphatase and tensin homolog (PTEN)deficient mice. J. Gastroenterol. Hepatol. 22(Suppl 1), S96-S100 (2007).

63. Chen, W. T. et al. Liver-specific knockout of GRP94 in mice disrupts cell adhesion, activates liver progenitor cells, and accelerates liver tumorigenesis. Hepatology 59, 947-957 (2014)

64. Moriya, K. et al. The core protein of hepatitis $C$ virus induces hepatocellular carcinoma in transgenic mice. Nat. Med. 4, 1065-1067 (1998).

65. Inokuchi, S. et al. Disruption of TAK1 in hepatocytes causes hepatic injury, inflammation, fibrosis, and carcinogenesis. Proc. Natl Acad. Sci. USA 107, 844-849 (2010).

66. Chisari, F. V. et al. A transgenic mouse model of the chronic hepatitis B surface antigen carrier state. Science 230, 1157-1160 (1985).

67. $Y e, H$. et al. Synergistic function of Kras mutation and HBx in initiation and progression of hepatocellular carcinoma in mice. Oncogene 33, 5133-5138 (2014).

68. Thorgeirsson, S. S. \& Santoni-Rugiu, E. Transgenic mouse models in carcinogenesis: interaction of c-myc with transforming growth factor alpha and hepatocyte growth factor in hepatocarcinogenesis. Br. J. Clin. Pharmacol. 42, 43-52 (1996).

69. Tönjes, R. R. et al. Autocrine mitogen lgEGF cooperates with c-myc or with the Hcs locus during hepatocarcinogenesis in transgenic mice. Oncogene 10, 765-768 (1995).

70. Calvisi, D. F. et al. Activation of the canonical Wnt/beta-catenin pathway confers growth advantages in C-Myc/E2F1 transgenic mouse model of liver cancer. J. Hepatol. 42, 842-849 (2005).

71. Klocke, R. et al. Lack of p53 accelerates hepatocarcinogenesis in transgenic mice constitutively overexpressing c-myc in the liver. FASEB J. 15, 1404-1406 (2001).

72. Katz, S. F. et al. Disruption of Trp53 in livers of mice induces formation of carcinomas with bilineal differentiation. Gastroenterology 142, 1229-1239 e3 (2012).
73. Lou, D. Q. et al. Conditional hepatocarcinogenesis in mice expressing SV 40 early sequences. Cancer Lett. 229, 107-114 (2005).

74. Lee, J. S. et al. Application of comparative functional genomics to identify bestfit mouse models to study human cancer. Nat. Genet. 36, 1306-1311 (2004).

75. Colnot, S. et al. Liver-targeted disruption of Apc in mice activates beta-catenin signaling and leads to hepatocellular carcinomas. Proc. Natl Acad. Sci. USA 101, 17216-17221 (2004)

76. Lee, G. H., Merlino, G. \& Fausto, N. Development of liver tumors in transforming growth factor alpha transgenic mice. Cancer Res. 52, 5162-5170 (1992).

77. Harada, N. et al. Hepatocarcinogenesis in mice with beta-catenin and Ha-ras gene mutations. Cancer Res. 64, 48-54 (2004).

78. Chen, X. \& Calvisi, D. F. Hydrodynamic transfection for generation of novel mouse models for liver cancer research. Am. J. Pathol. 184, 912-923 (2014).

79. Calvisi, D. F. et al. Increased lipogenesis, induced by AKT-mTORC1-RPS6 signaling, promotes development of human hepatocellular carcinoma. Gastroenterology 140, 1071-1083 (2011).

80. Tward, A. D. et al. Distinct pathways of genomic progression to benign and malignant tumors of the liver. Proc. Natl Acad. Sci. USA 104, 14771-14776 (2007).

81. Lee, S. A. et al. Integration of genomic analysis and in vivo transfection to identify sprouty 2 as a candidate tumor suppressor in liver cancer. Hepatology 47, 1200-1210 (2008).

82. Shang, N. et al. Focal adhesion kinase and beta-catenin cooperate to induce hepatocellular carcinoma. Hepatology 70, 1631-1645 (2019).

83. Ju, H. L. et al. Investigation of oncogenic cooperation in simple liver-specific transgenic mouse models using noninvasive in vivo imaging. PLOS ONE $\mathbf{8}$, e59869 (2003).

84. Wang, C. et al. Inactivation of Spry2 accelerates AKT-driven hepatocarcinogenesis via activation of MAPK and PKM2 pathways. J. Hepatol. 57, 577-583 (2012).

85. Ju, H. L., Han, K. H., Lee, J. D. \& Ro, S. W. Transgenic mouse models generated by hydrodynamic transfection for genetic studies of liver cancer and preclinical testing of anti-cancer therapy. Int J. Cancer 138, 1601-1608 (2016).

86. Wangensteen, K. J. et al. A facile method for somatic, lifelong manipulation of multiple genes in the mouse liver. Hepatology 47, 1714-1724 (2008).

87. $\mathrm{Xu}, \mathrm{C}$. R. et al. Bmi1 functions as an oncogene independent of Ink4A/Arf repression in hepatic carcinogenesis. Mol. Cancer Res. 7, 1937-1945 (2009).

88. Che, L. et al. Cholesterol biosynthesis supports the growth of hepatocarcinoma lesions depleted of fatty acid synthase in mice and humans. Gut 1, 566-578 (2019).

89. Shang, N. et al. Focal adhesion kinase and beta-catenin cooperate to induce hepatocellular carcinoma. Hepatology 5, 1631-1645 (2019).

90. Chen, S., Cao, Q., Wen, W. \& Wang, H. Targeted therapy for hepatocellular carcinoma: challenges and opportunities. Cancer Lett. 460, 1-9 (2019).

91. Tao, J. et al. Activation of beta-catenin and Yap1 in human hepatoblastoma and induction of hepatocarcinogenesis in mice. Gastroenterology 147, 690-701 (2014)

92. Li, X. et al. Co-activation of PIK3CA and Yap promotes development of hepatocellular and cholangiocellular tumors in mouse and human liver. Oncotarget 6, 10102-10115 (2015).

93. Fan, L. et al. Bmi1 is required for hepatic progenitor cell expansion and liver tumor development. PLOS ONE 7, e46472 (2012).

94. Zhou, L., Huang, Y., Li, J. \& Wang, Z. The mTOR pathway is associated with the poor prognosis of human hepatocellular carcinoma. Med. Oncol. 27, 255-261 (2010).

95. Li, L. et al. Inactivation of fatty acid synthase impairs hepatocarcinogenesis driven by AKT in mice and humans. J. Hepatol. 64, 333-341 (2016).

96. Schlaeger, C. et al. Etiology-dependent molecular mechanisms in human hepatocarcinogenesis. Hepatology 47, 511-520 (2008).

97. Juric, V. et al. Monocytes promote liver carcinogenesis in an oncogene-specific manner. J. Hepatol. 64, 881-890 (2016).

98. van der Windt, D. J. et al. Neutrophil extracellular traps promote inflammation and development of hepatocellular carcinoma in nonalcoholic steatohepatitis. Hepatology 68, 1347-1360 (2018).

99. Matter, M. S. et al. Oncogenic driver genes and the inflammatory microenvironment dictate liver tumor phenotype. Hepatology 63, 1888-1899 (2016). 